\title{
DESIGN AND DEVELOPMENT OF WRIST-TILT BASED PC CURSOR CONTROL USING ACCELEROMETER
}

\author{
Ankush Nayyar ${ }^{1}$, Hemant Lenka $^{2}$ \\ ${ }^{1,2}$ Centre for Development of Advanced Computing \\ nayyar2720gmail.com \\ hemant_lenka@yahoo.com
}

\begin{abstract}
Human computer Interfacing apparatus is key part in modern electronics period. Motion recognition can be well introduced in present day computers to play games. In this work simple inertial navigation sensor like accelerometer can be use to get Dynamic or Static acceleration profile of movement to move cursor of mouse or even rotate 3-D object. In this paper a human computer interfaces system is presented, which will be able to act as an enhanced version of one of the most common interfacing system, which is computer mouse. In this research work, an alternative to interact with computer, for those who do not want to use conventional HCI (human computer interface) or not able to use conventional human computer interface and this achieved by using a sensor accelerometer mount on human wrist or anywhere in human body. Accelerometer device use to detect the position in $x, y$ direction caused by movement of device mount on the wrist, as referred from acceleration of gravity $(1 \mathrm{~g}=9.8 \mathrm{~m} / \mathrm{s} 2)$. Accelerometer is connected with PIC16F877A for analog to digital conversion and PIC16F877A are connected with LCD for displaying the co-ordinates $(x, y)$ in which the accelerometer move and it further connected with ZigBee transmitter which is used to transmit the wireless signal to ZigBee receiver. ZigBee receiver receives the signal from transmitting end and transferred to PC through MAX232 serial communication. In PC an application for cursor control in response to accelerometer movement is developed.
\end{abstract}

\section{KEYWORDS}

Accelerometer, PIC Microcontroller, ZigBee Protocol

\section{INTRODUCTION}

The core aim behind the project is to be able to sense the movement of a user hand and use it to control the movement of mouse on screen, which is very useful for gaming and for bodily challenged persons. For sensing hand movement an accelerometer device is used. The need of this research work is to investigate the possibility of creating a wireless mouse that could be used by anyone, anywhere, without having a flat surface to move it and also without holding mouse in hand, for this reason, a device accelerometer used to detect its own orientation and ZigBee used to transmit or receiving data wirelessly so that there would not need to be any wires connection, which limit the range and comfort.

Accelerometer is a device that senses the tilt in any direction (x,y,z) and the output of device is usually analog. This output is proportional to the rate of acceleration vary and acceleration are directly proportional to the rate at which device velocity change and velocity are speed and direction, so there is change in speed and direction there is acceleration. If there is force act on a body or object there is change in speed or direction and if the force is zero then the velocity of

DOI : 10.5121/ijcsea.2013.3407 
object is constant and acceleration become zero. In this research an accelerometer are use to sense the tilt in two direction $(\mathrm{x}, \mathrm{y})$ and there co-ordinates are display on LCD using PIC controller programming in embedded c. ZigBee tans-receiver are used to transmit and receive the signal over air.PIC microcontroller are also used to convert the analog signal to digital receiving from accelerometer through inbuilt ADC.TTL to RS232 line driver (max232) are used for interfacing microcontroller with PC because microcontroller is TTL compatible and pc is CMOS compatible. This is a simple low cost; low power inertial sensor based mouse with wireless capability will offer ease of use. It can be use in Gesture Recognition.

\section{RELATED WORK}

The focal aim of this project is to present the basic needs for physically challenged people like food, water and medicine and to control the electrical devices like Fan, bulb by using switch buttons and also for controlling wheel chair by using MEMS technology (micro electro mechanical sensor), which is an extremely sensitive sensor capable of detecting the tilt. This device is convenient and this system operation is entirely driven by wireless technology. Client can wear the MEMS to his finger and can control it by tilting up, down, left and right side respectively. This system is also makes use of Microcontroller like LPC2148. This project also makes use of switches which are operate by physically challenged persons to control the electrical appliances like fan, bulb and some other devices Using AT89S52 Microcontroller and wireless technology like ZigBee.[1]

This paper presents an accelerometer-based digital pen designed for handwritten digit and gesture trajectory recognition applications. This pen consists of a 3-axial accelerometer, a microcontroller, and an RF wireless transmission module for sensing and collecting accelerations of handwriting. The investigational consequences have successfully validated the success of the trajectory detection algorithm for handwritten digit and gesture recognition using the proposed product. [2]

In this research work a health monitoring of human physiological signals such as temperature and pulse using ZigBee is formed in this work, by this device the temperature and pulse of humans can be observe from a distant location, and some abnormalities can be easily indicated via SMS .The measurements obtain from the temperature sensor and heart beat sensor are send to the PC through ZigBee module. The PC collects the information and also sends SMS, to the indicated mobile number through a GSM module. [3]

Numerous responsibilities that are perform on the computer use of both a keyboard on the mouse and a lot of people find it frustrating and painful to have to switch back and forth between them. This proposed work is a great solution for this. The only two major changes to the mouse since its beginning and these changes will be more buttons, and interface changes (PS/2, USB, and Wireless) but the interface method are not changed. The touch pad mouse as well as the stick mouse have been proposed for a laptop, but have not gained momentum outside of laptops and many laptop users usually carry a USB mouse with the laptop. The proposed accelerometer based mouse can be treated as the new times input tool. This proposed device is extra accepted in its feel and provides the user with better ease of use. [4]

\section{BLOCK DIAGRAM}

This block diagram consist two module transmitting and receiving shown in figure1. In this project a wireless human computer interfaces for controlling the computer mouse cursor is developed. Transmitting module divided in different unit, power supply unit, microcontroller unit, LCD (16*2) display unit, accelerometer and transmitting unit and receiving part is an integration of power supply, receiving unit, MAX 232, DB9 connecter. 


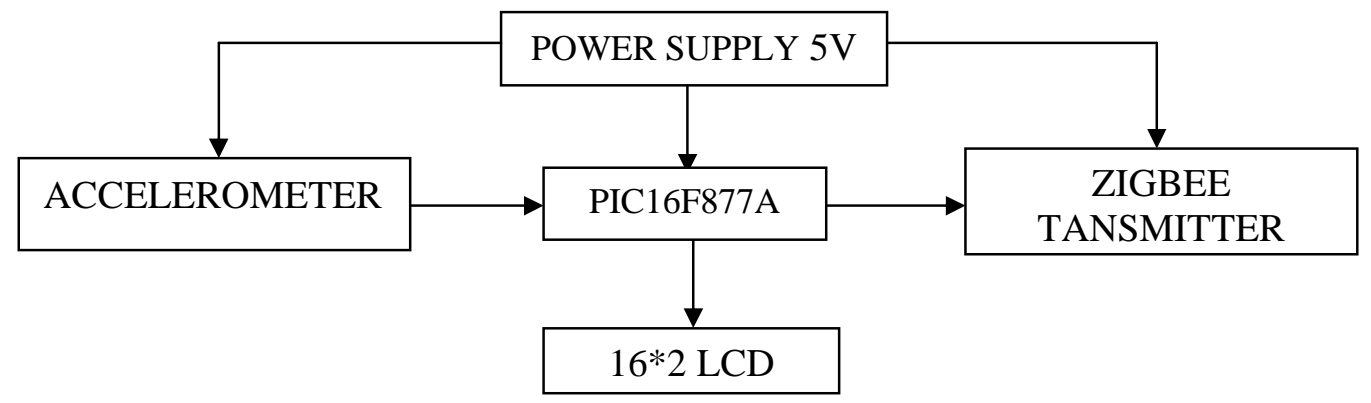

Figure 1. Block diagram of transmitting unit.

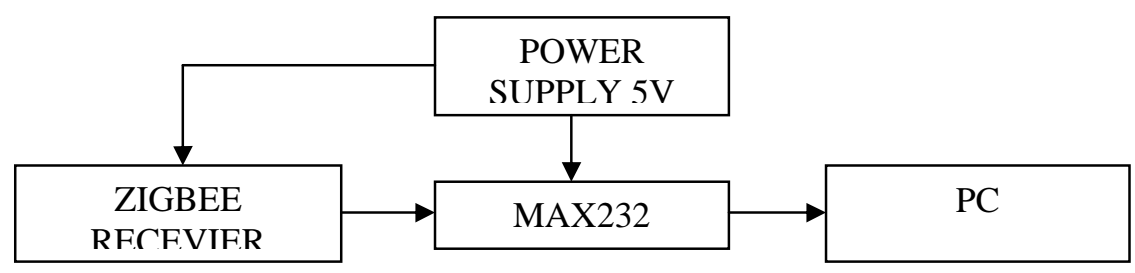

Figure 2. Block diagram of receiving unit

In transmitting part accelerometer can be used to get the movement of user wrist to move cursor of mouse or Gyroscope to even rotate 3-D object. Accelerometer sensor are used measures the level of accelerometer where it is mounted this enable us to measure tilt of a platform with respect to earth axis, and then analog output of accelerometer in $\mathrm{x}, \mathrm{y}$ plane is applied to microcontroller(PIC16f877A), which is used to control the display on LCD and also connected with ZigBee transmitter. Microcontroller is energized by $5 \mathrm{~V}$ power supply. LCD is used to display co-ordinate in which the cursor movement takes place and the ZigBee transmitter are used to transmit the signal to receiver module which receive the signal send by transmitter and then this signal is applied to MAX232. MAX232 is used while interfacing microcontroller with PC to verify the Baud rate and changes the voltage level because microcontroller is TTL friendly, whereas PC is CMOS friendly. MAX232 connect the microcontroller and PC through female DB9 pin. In PC an application is developed which is use to operate the mouse in response to the accelerometer tilt.

\subsection{Accelerometer:}

In this research accelerometer 1156 is used. Accelerometer sensor used to measure static (earth gravity) or dynamic acceleration in all three axis shows in Figure 3. And Figure 4. Accelerometer sensor dealings with level of acceleration where it is mounted, this make possible to determine acceleration or deceleration of objects or tilt of a stage with respected to earth gravity. Accelerometer Sensor provides zero G output which detects linear free fall. Acceleration is a vector quantity which has the direction and measured in meters per second. Gravitational acceleration produced on all objects on earth due to earth gravity and therefore by monitoring the axis of object it is easy to find the level of tilt. Accelerometer interfacing to microcontroller unit just needs to give supply ( $+5 \mathrm{v}$ and $\mathrm{Gnd}$ ) to the sensor and then it gives analog signals from pin $\mathrm{X}$, $\mathrm{Y}$ and $\mathrm{Z}$ corresponds to its orientation with respect to Earth. At zero gravity the output of axis ( $\mathrm{x}$, $\mathrm{y}, \mathrm{z})$ is $1.65 \mathrm{~V}$, and output of accelerometer on sensitivity range $1.5 \mathrm{~g}$ is $800 \mathrm{mV} / \mathrm{g}$ and on sensitivity range $6 \mathrm{~g}$ is $200 \mathrm{mV} / \mathrm{g}$. 


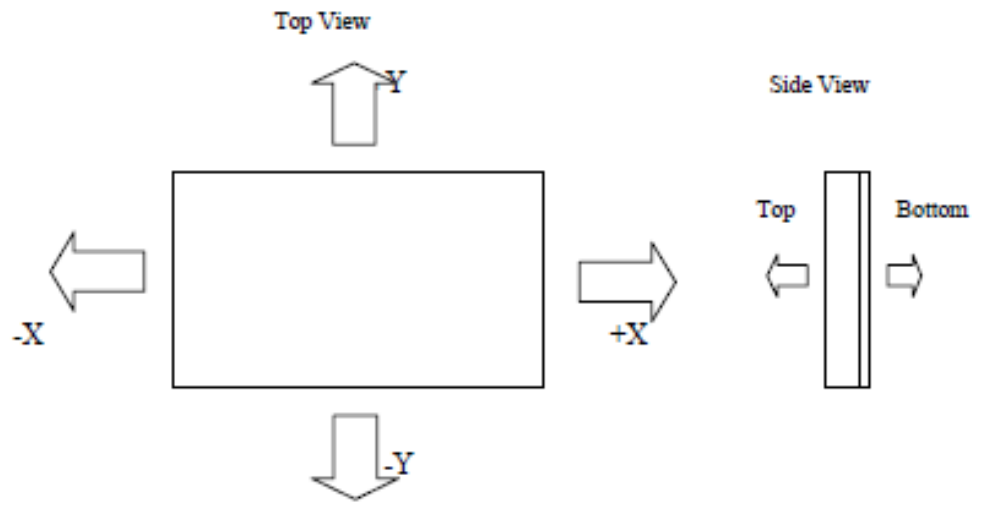

Figure 3. Dynamic Acceleration Movement

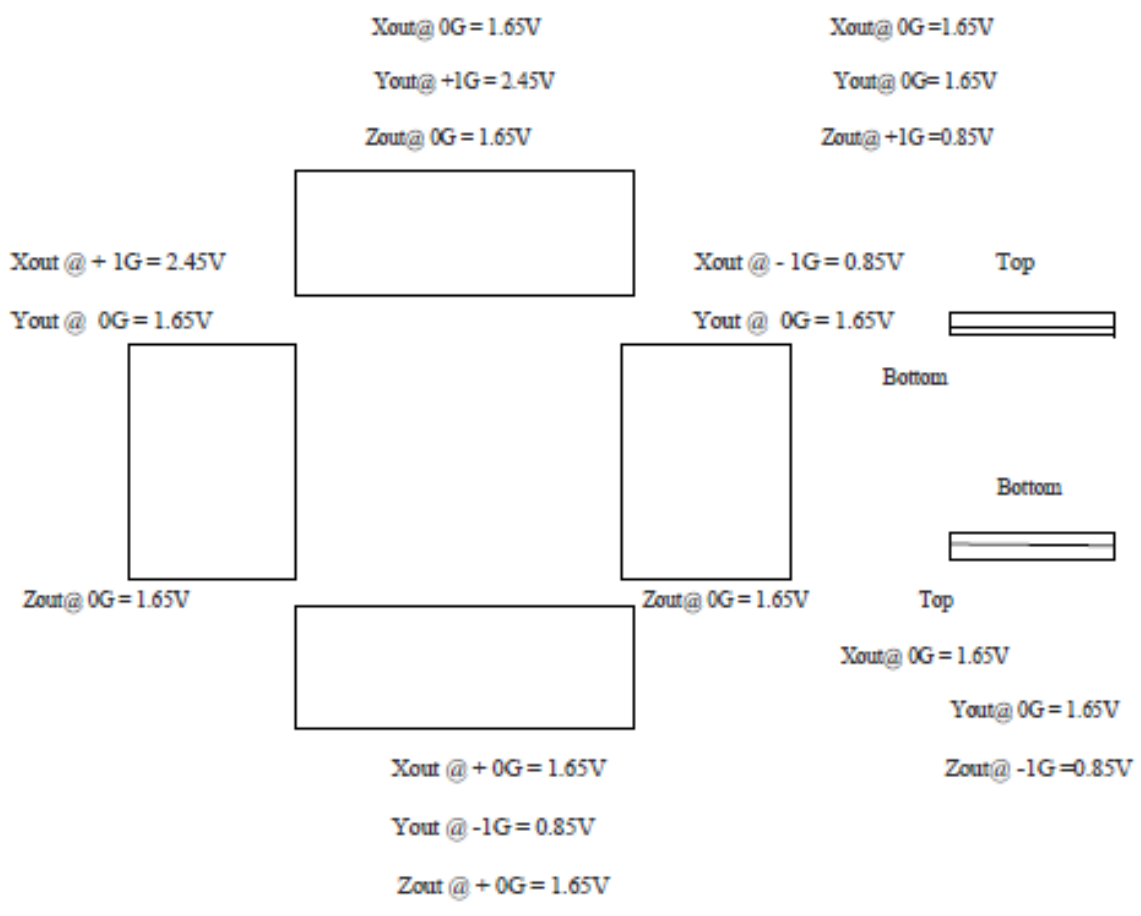

Figure 4. Static Acceleration Movemenet

\subsection{Transmitting and receiving unit (ZigBee)}

In Tx and Rx units ST1197 Serial Interface IC for RF Transceiver based on CC1100/CC2500 is used. ST1197 Serial Interface IC supports any RF based modules/transceiver which is based on Texas Instrument's Chipcon ICs like CC1100 (433 MHz) and CC2500 (2.4 GHZ). This will provides a easy UART interface for transmission and reception of serial data at the rate $9600 \mathrm{bps}$ (baud per second). This can be used for applications where two way wireless data transmission are needed. Communication protocol is controlled itself and completely transparent to user interface. The IC can be embedded to this current design so that wireless communication can be set up easily. This IC works in half-duplex mode that is either in transmitting or receiving but not both at same time. After every transmission, IC switched back to receiving mode automatically. The LED indicates whether IC is currently receiving or transmitting data. The data checked for 
CRC error if any. The IC has 64 bytes of buffer for arriving data. while you power on the unit, the TX LED will blink representing that initialization is complete and detect the Chipcon based transceiver. If LED remains continuous on then the problem could be related to connection between IC and RF Module. RF Trans-receiver with $2.4 \mathrm{GHz}$ frequency and 30 meters range model 3478 is used with ST1197. In a usual system, this type of trans-receiver will be used with a microcontroller. It provides wide hardware support for packet handling, data buffering, burst transmissions. RF Module and ST1197 are working below at 3V level.

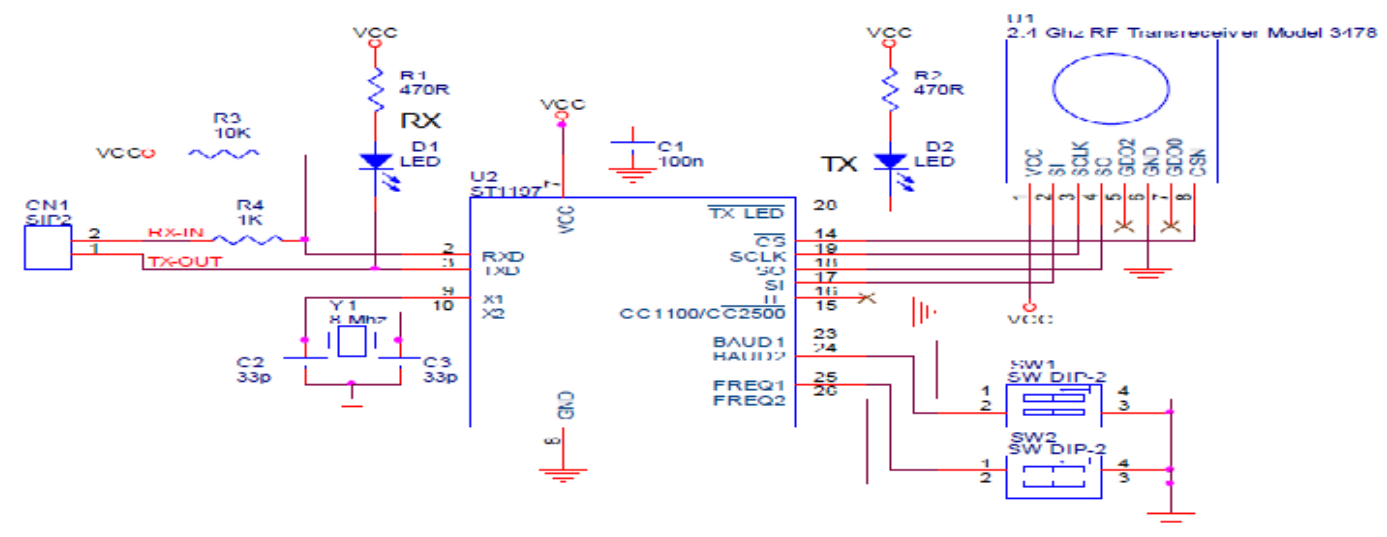

Figure 5. Shows internal schematic of ZigBee

\section{Baud Rate Setting}

BAUD1 and BAUD2 pins of ST1197 serial interface IC are used for baud rate setting. These pins are default HIGH if it is left unconnected. During power up mode these pin status is read only and any changes to these pin during operation will have no effect. Baud rate setting for ST1197 IC shown in Table 1. [5]

\begin{tabular}{|c|c|c|}
\hline BAUD 1 & BAUD 2 & MODE \\
\hline HIGH & HIGH & $9600 \mathrm{bps}$ \\
\hline LOW & HIGH & $4800 \mathrm{bps}$ \\
\hline HIGH & LOW & $38400 \mathrm{bps}$ \\
\hline LOW & LOW & $19200 \mathrm{bps}$ \\
\hline
\end{tabular}

\section{Frequency Channel Setting}

Table 1. Baud Rate Setting

FREQ1 and FREQ2 pins will be default high if it is left unconnected. These pins are in read only status during power up. The purpose of Setting Frequency Channel is that it can be used to have multiple sets of operating at same time but without any interfering. The pair having similar Channel setting will be capable to communicate with each other. Channel setting shown in Table 2. [5] 
International Journal of Computer Science, Engineering and Applications (IJCSEA) Vol.3, No.4, August 2013

\begin{tabular}{|c|c|c|}
\hline FREQ 1 & FREQ 2 & MODE \\
\hline HIGH & HIGH & Channel 1 \\
\hline LOW & HIGH & Channel 2 \\
\hline HIGH & LOW & Channel 3 \\
\hline LOW & LOW & Channel 4 \\
\hline
\end{tabular}

Table 2. Frequency Channel Setting

\section{SOFTWARE METHODOLOGY}

Figure 6. Shows the software methodology of the co-ordinates displays on LCD screen for this mikro c software are used and programming is done in embedded c. As shown Figure 6. First of all check for tilt detection if there is no tilt in accelerometer then mouse cursor is in initial position and LCD display the name of project if there is tilt in accelerometer then the output of accelerometer axis is send to microcontroller where an embedded c code is burn in microcontroller for co-ordinate display on LCD screen. After that accelerometer tilt position given to ZigBee transmitter, ZigBee transmitter transmit the signal wirelessly, which is received by ZigBee receiver and given to PC using MAX232 serial communication and DB9 female cable are used to connect with PC. In PC side an application to control cursor of mouse in answer to the tilt pose of accelerometer are designed by using visual basic programming.

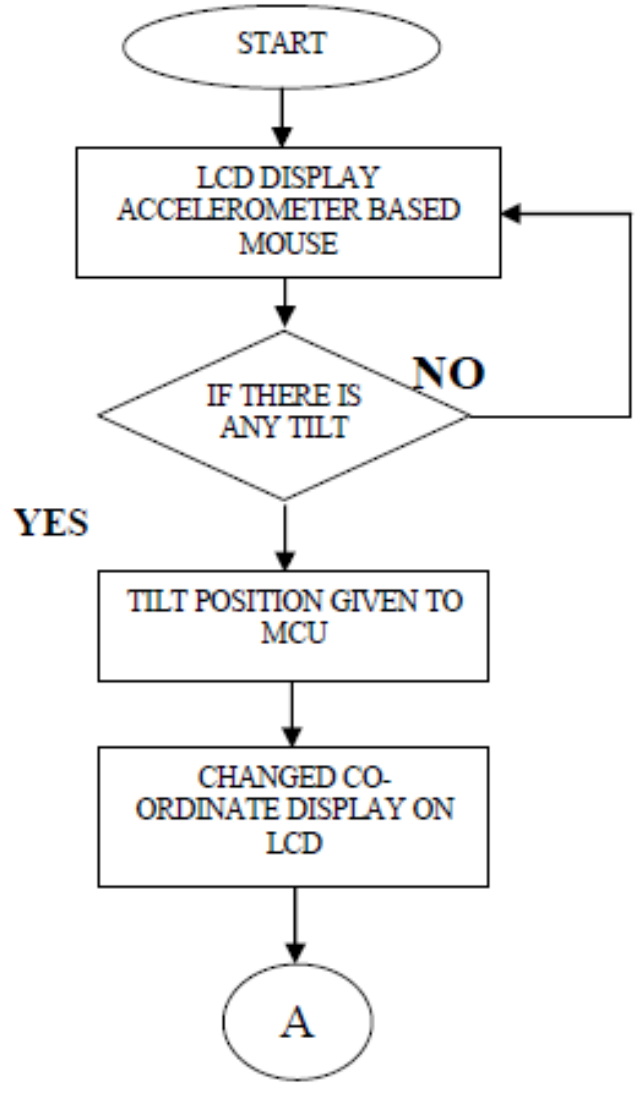




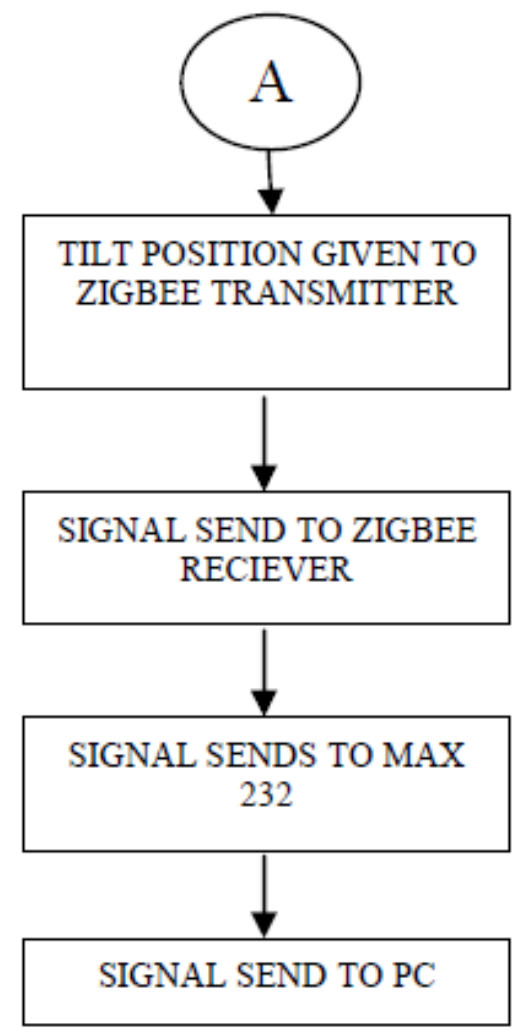

Figure 6. Flow Chart for cursor control

\section{RESULT}

Accelerometer device used to detect the static or dynamic change in position. If there is some tilt in accelerometer then cursor position changes in response to that tilt and position of x,y coordinate and position of mouse cursor on computer screen that is left, right, up, down direction are shown on LCD shown in Figure 7. and Figure 8.

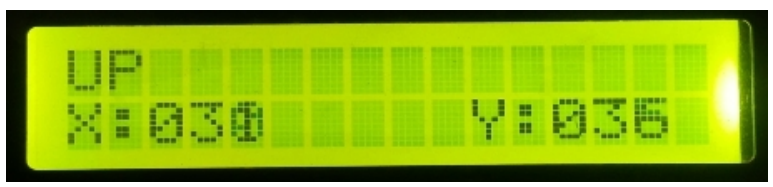

Figure 7. Shows the Direction and Co-ordinates of Cursor

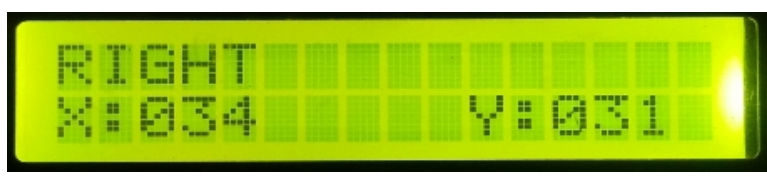

Figure 8. Shows the Direction and Co-ordinates of Cursor

\section{CONCLUSION}

This design is used to control computer mouse cursor for this an accelerometer sensor is used. The advantages of using an accelerometer approach intended for control using laryngeal 
vibrations, compared to supplementary sensor, is that the user has only control over an apparatus or device. Surroundings noise is not recorded and consequently it does not interfere with a user controlling an instrument or device.

The scope this project is that we can increase the communication speed between transmitter and receiver and also the mouse movement convert in $3 \mathrm{D}$ environment using $\mathrm{z}$ axis. This project is also useful for gaming and for monitoring disable people.

\section{REFERENCES}

[1] B. Ramana Reddy, J. V. Sharma, "Movement based and Voice enabled Device Switching for basic needs of Physically Challenged Persons" International Journal of Science and Research (IJSR), India Online ISSN: 2319-7064, Volume 2 Issue 3, March 2013

[2] Jeen-Shing Wang, and Fang-Chen Chuang .An Accelerometer-Based Digital Pen With a Trajectory Recognition Algorithm for Handwritten Digit and Gesture Recognition IEEE Transactions on Industrial Electronics VOL. 59, NO. 7, JULY 2012.

[3] S.Josephine Selvarani, Online Health Monitoring System Using Zigbee, “ International Journal on Computer Science and Engineering (IJCSE)” ISSN : 0975-3397 Vol. 3 No. 4 Apr 2011

[4] Manav Kataria, Vinu Bhaskar, Abhishek Sharma, Mizan Abraha "ACCELEROMETER MOUSE" (2011, 04). Air Mouse. StudyMode.com. Retrieved 04, 2011.

[5] www.sunrom.com

[6] Tiexiang Wen; Lei Wang; Jia Gu; Bangyu Huang, "A 3-D Acceleration-Based Control Algorithm for Interactive Gaming Using a Head-Worn Wireless Device," Bioinformatics and Biomedical Engineering , 2009. ICBBE 2009. 3rd International Conference on , vol., no., pp.1,3, 11-13 June 2009

[7] Piedrahita,; Andres, Giovanny; Guayacundo,; Marcela, Diana, "Evaluation of Accelerometers as Inertial Navigation System for Mobile Robots," Robotics Symposium, 2006. LARS '06. IEEE 3rd Latin American, vol., no., pp.84,90, 26-27 Oct. 2006

[8] Lombardi, A.; Ferri, M.; Rescio, G.; Grassi, M.; Malcovati, P., "Wearable wireless accelerometer with embedded fall-detection logic for multi-sensor ambient assisted living applications," Sensors, 2009 IEEE , vol., no., pp.1967,1970, 25-28 Oct. 2009

[9] Sa-kwang Song; Jaewon Jang,; Soojun Park, "A Phone for Human Activity Recognition Using Triaxial Acceleration Sensor," Consumer Electronics, 2008. ICCE 2008. Digest of Technical Papers. International Conference on , vol., no., pp.1,2, 9-13 Jan. 2008

\section{AUTHORS}

Short Biography

Ankush Nayyar received his B.Tech. Degree in ECE from IGCE, Punjab Technical University, India, in 2010. He is currently pursuing the M.Tech. Degree at the Centre for development of advanced computing mohali, India, with a research topic "Design and Development of Wrist-Tilt Based PC Cursor Control Using Accelerometer."

Hemanta kumar Lenka(Engineer). He did his Degree in Electronics and Communication Engineering (A.M.I.E). He did his MTECH in IT (From Rajasthan Deemed University on July 2005). His area of interest is AutoCAD Auto lisp programming and Customisation.
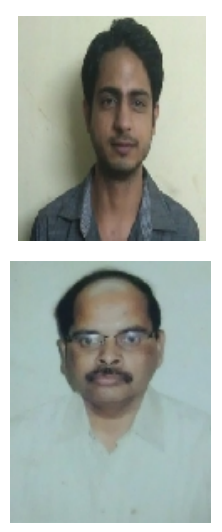